\title{
La simulation numérique révèle le passé tourmenté des galaxies
}

Frédéric Bournaud (Frederic.bournaud@cea.fr)

Service d'Astrophysique du CEA-Saclay, 91191 Gif-sur-Yvette

\section{Les simulations}

numériques sur

les supercalculateurs

modernes permettent de calculer l'évolution

des galaxies avec

une résolution qui

égale parfois celle des

plus grands télescopes.

Elles deviennent donc

un outil fondamental

de l'interprétation des

observations,

\section{en permettant}

de comprendre quels

mécanismes d'évolution

passés sont responsables

des propriétés observées

aujourd'hui dans les

galaxies. Et c'est souvent

\section{un passé complexe et}

plus tourmenté qu'on

aurait pu le croire qui

est ainsi révélé par les

modèles numériques.

(1) Le nombre limité de particules tend à induire une surestimation des interactions de paires entre corps proches par rapport aux interactions d'ensembles à grande distance ; les forces à courte distance sont donc atténuées dans les modèles, de telle sorte que l'évolution dynamique soit dominée par les interactions collectives à longue portée, comme dans les vraies galaxies.

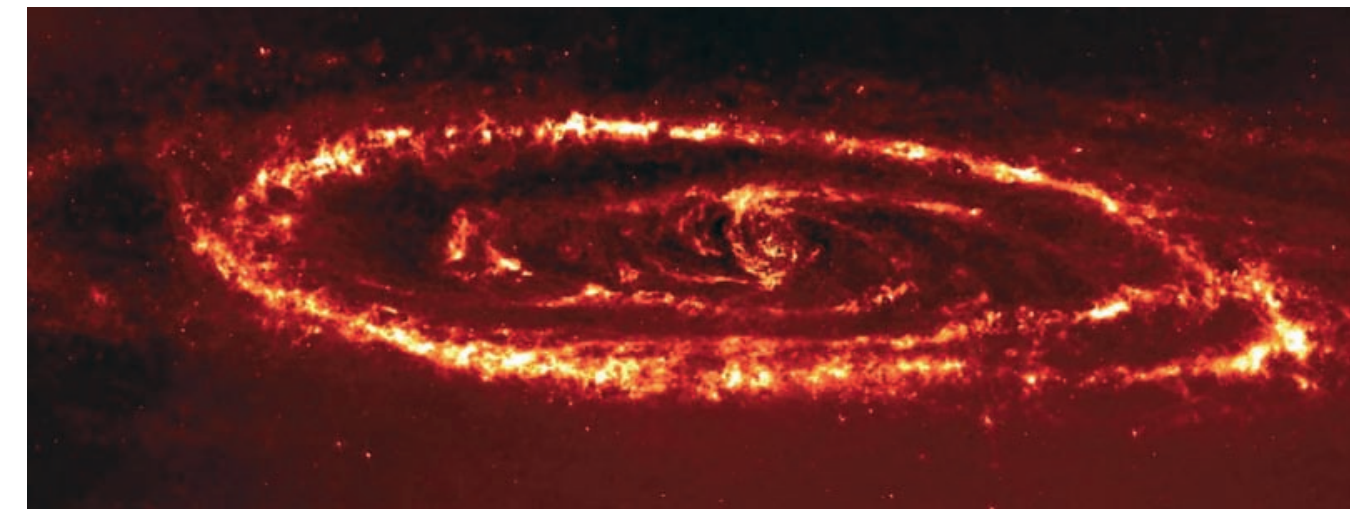

Cartographie de l'émission de poussières chaudes et de macromolécules de la galaxie d'Andromède par le satellite Spitzer.

Les premières galaxies formées dans l'Univers étaient des galaxies naines, qui se sont assemblées progressivement en des systèmes de plus en plus massifs, pour constituer finalement les grandes galaxies spirales que nous connaissons aujourd'hui, dont notre Voie Lactée. Selon le scénario de croissance hiérarchique, l'essentiel de ces collisions et fusions entre galaxies se serait produit dans la première moitié de la vie de l'Univers, soit en 6 ou 7 milliards d'années, la seconde partie étant notablement plus calme.

Néanmoins, les moyens modernes de simulations numériques nous aident maintenant à mieux comprendre et interpréter les images fournies par des télescopes de plus en plus performants. Ces modèles numériques ont révélé que le passé récent des galaxies, même au cours de leur dernier milliard d'années d'évolution, était souvent plus tourmenté qu'on ne le pensait. Inutile de chercher bien loin pour trouver des victimes : la galaxie d'Andromède, notre principale voisine et seule grande galaxie spirale visible à l'œil nu dans notre ciel, aurait, elle-même, été frappée en plein cœur, il y a 200 millions d'années.

\section{Simuler l'évolution des galaxies et leurs collisions}

La simulation numérique de galaxies est née à la fin des années 1970. Les premières simulations, de type "trois-corps restreint ", se contentaient de simuler le mouvement de quelques particules représentant les étoiles des régions externes d'une galaxie, autour d'une masse centrale dominante, représentant les régions internes les plus massives. Un modèle en apparence simpliste, mais qui a toutefois permis de comprendre quelques phénomènes cinématiques à l'œuvre dans les galaxies spirales.

Les supercalculateurs modernes permettent des modélisations beaucoup plus réalistes (voir encadré, p. 8) : la centaine de milliards d'étoiles que contient une galaxie spirale est reproduite par plusieurs millions de particules, la loi de la gravité Newtonienne étant modifiée aux courtes distances pour compenser l'effet de cette différence de nombre ${ }^{(1)}$. La gravité de chaque particule est prise en compte. Le gaz interstellaire et sa dynamique dissipative sont également simulés, soit par des codes hydrodynamiques, soit en modélisant les collisions inélastiques entre les nuages de gaz interstellaire. La formation de nouvelles étoiles dans ce gaz est, elle aussi, reproduite par les simulations, qui atteignent depuis quelques années un certain degré de raffinement dans la prise en compte de phénomènes secondaires : perte de masse des étoiles, libération d'énergie par les explosions de supernovae, chauffage et refroidissement du gaz... Le tout avec des résolutions qui peuvent atteindre quelques dizaines de parsecs, soit aussi bien qu'avec de grands télescopes sur les galaxies les plus proches de la nôtre dans l'Univers Local : de quoi comparer en détail modèles numériques et observations de vraies galaxies. Ces simulations numériques peuvent résoudre la dynamique interne des galaxies très en détail dans les régions centrales, mais elles sont tout aussi performantes pour modéliser les 
Figure 1 : Simulation numérique de la collision de deux galaxies spirales, vues initialement (à gauche) puis 300 millions d'années plus tard (à droite). Les deux galaxies fusionnent en une galaxie elliptique (sphéroïdale), mais de grands lambeaux de matière leur sont arrachés par les forces de marée. Les couleurs mettent ici en évidence des différences de composition : en orange, les régions dominées par les vieilles étoiles, en bleu les régions riches en gaz interstellaire et étoiles jeunes. Chaque disque galactique a initialement un diamètre de 50 kiloparsecs (environ 200000 années-lumière).

Figure 2 : Cartographie de l'émission de poussières chaudes et de macromolécules de la galaxie d'Andromède par le satellite Spitzer. L'anneau externe était connu depuis une vingtaine d'années, mais l'anneau interne avait jusqu'ici échappé aux observations dans d'autres longueurs d'ondes. Le décentrage respectif des anneaux par rapport au centre de la galaxie ne peut s'expliquer que s'ils résultent d'une collision passée - sans doute avec le petit compagnon $\mathrm{M} 32$
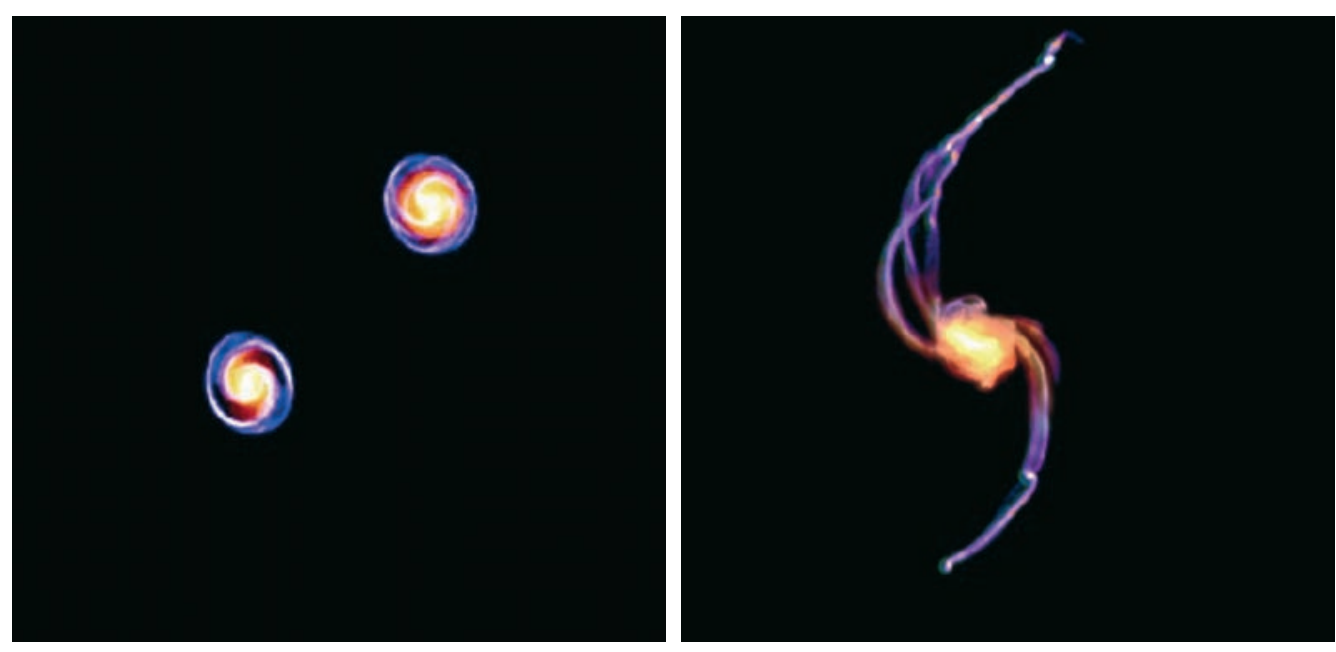

effets de l'interaction entre galaxies voisines et les collisions (fig. 1).

\section{Les malheurs de la galaxie d'Andromède}

Le satellite Spitzer, à l'aide de sa caméra infrarouge IRAC, a récemment cartographié l'émission de la poussière chaude de la galaxie d'Andromède. L'essentiel de la masse du milieu interstellaire des galaxies (hors matière " noire ") est constituée de gaz atomique et moléculaire observable par les radiotélescopes, mais avec une résolution limitée. La poussière permet de tracer beaucoup plus finement les structures du milieu interstellaire. Les observations de Spitzer ont révélé, en plus des bras spiraux d'un anneau externe déjà connu, la présence d'un anneau interne jusqu'ici masqué par la forte luminosité du bulbe central (fig. 2). Deux mécanismes peuvent expliquer la présence d'un système d'anneaux concentriques dans le disque d'une galaxie : soit l'évolution interne et l'interaction entre une barre centrale et les bras spiraux, soit la collision de plein fouet avec une autre galaxie.

Des simulations numériques ont permis d'étudier les deux mécanismes et de montrer que l'évolution interne ne peut expliquer les propriétés des anneaux de la galaxie d'Andromède, par exemple leur taille relative. À l'opposé, ces mêmes simulations montrent qu'une collision de galaxies peut parfaitement reproduire la morphologie observée [1] (fig. 3). En suggérant que le coupable est probablement la petite galaxie M32 encore visible aujourd'hui non loin d'Andromède, les simulations ont même permis de reconstituer son orbite passée. M32 était, il y a peu, une petite galaxie spirale dont on ne voit plus que le bulbe aujourd'hui. Son disque externe lui a été arraché lorsqu'elle a percuté de plein fouet le disque bien plus grand de la galaxie d'Andromède, presque en son centre ; sa masse actuelle n'est plus qu'un vingtième de celle d'Andromède, contre le dixième avant la collision. La collision a en même temps créé un système d'anneaux en expansion dans la galaxie d'Andromède (un peu comme une pierre jetée dans l'eau) que l'on peut encore observer aujourd'hui. En effet, d'après les simulations, la collision se serait produite il y a 200 millions d'années : c'est peu à l'échelle des galaxies, à peine le temps que le disque d'Andromède met pour faire un tour ; c'est même un âge relativement court à l'échelle de la Terre, puisque c'est l'époque des dinosaures.

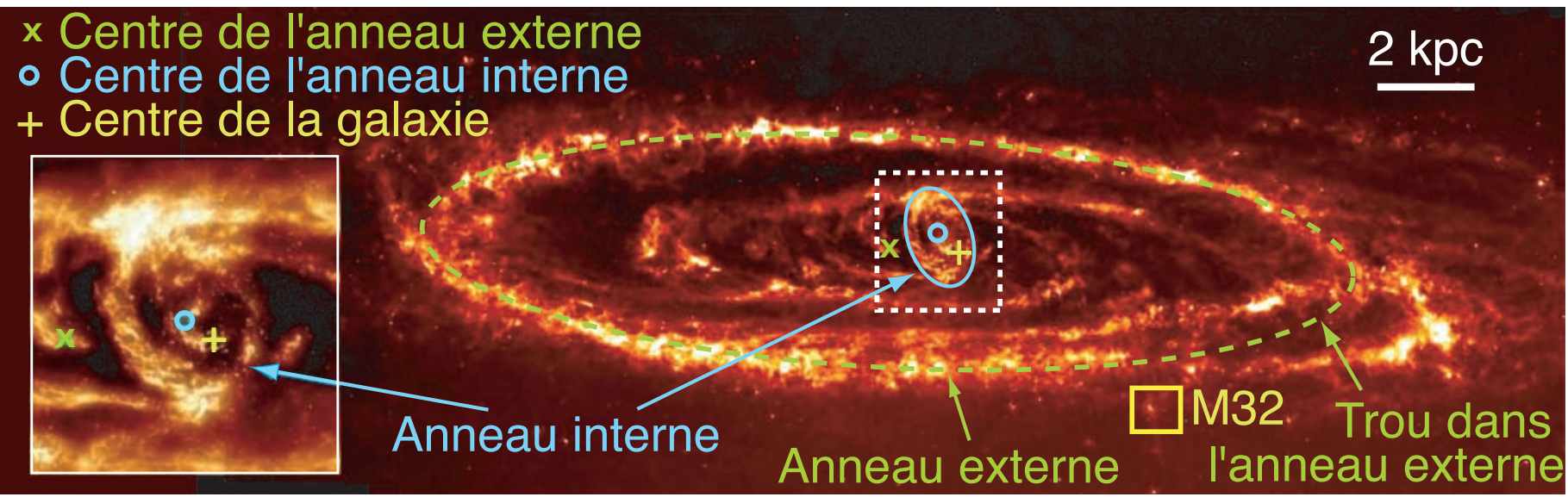



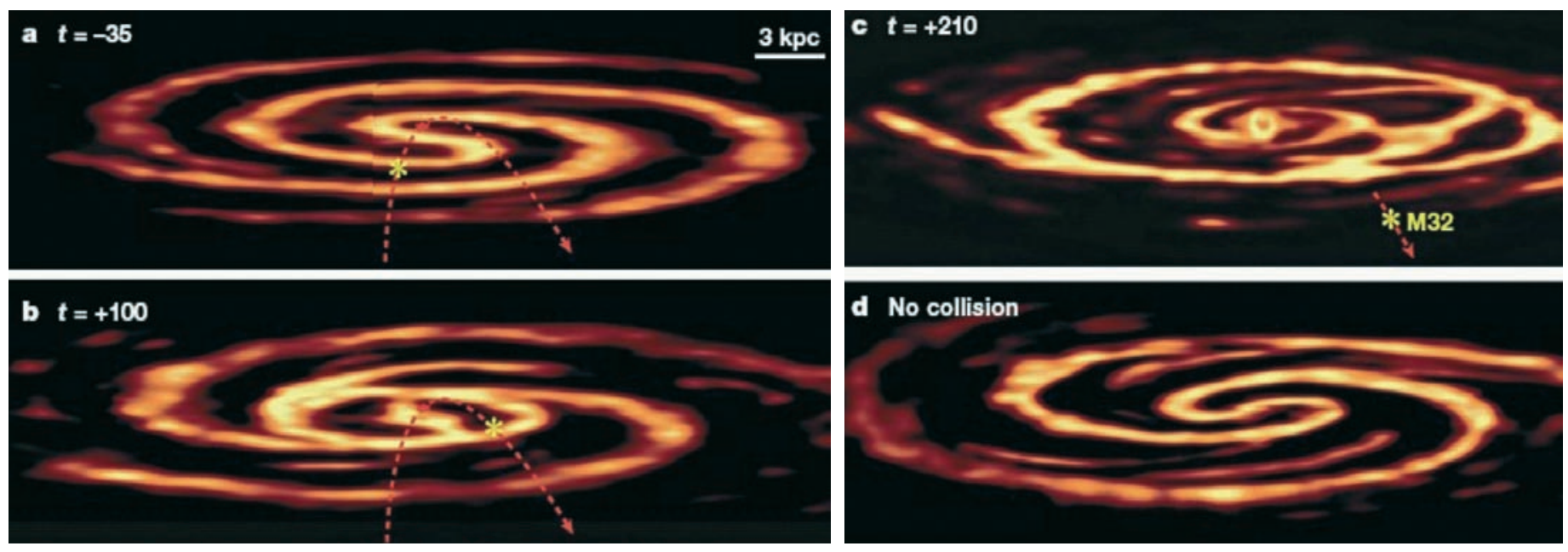

\section{Surprenante galaxie du Coquillage}

La petite galaxie M32 a laissé des traces, mais n'a pas complètement détruit le disque de la galaxie d'Andromède. Des phénomènes beaucoup plus intenses peuvent se produire lors des collisions entre grandes galaxies spirales de masses comparables. Les forces de marée(2) mises en jeu peuvent déformer les galaxies, et leur arracher de grandes quantités de matière sous la forme de longs filaments s'étendant dans le milieu intergalactique (fig. 1).

Un cas particulièrement intrigant est celui de la galaxie « du Coquillage », NGC5291. Elle a subi, comme Andromède, une collision frontale, une autre galaxie l'ayant traversée en son centre. Mais, cette fois, l'autre galaxie était bien plus massive que M32. Le changement rapide des forces lors de la collision gravitationnelle a expulsé les régions externes du disque, dont on ne voit plus aujourd'hui qu'un gigantesque anneau de gaz étendu sur 150 kiloparsecs une dizaine de fois plus grand que le disque de la galaxie initiale. Au milieu ne demeurent plus que les régions centrales de la galaxie progénitrice, devenue galaxie elliptique sous l'effet de la collision (figure 4, cette galaxie est initialement vue sous la forme d'un disque bleuté sur la figure 5). La galaxie qui l'a percutée est, quant à elle, repartie à grande vitesse (de l'ordre de $1000 \mathrm{~km} / \mathrm{s}$ ) sans subir de bouleversements majeurs.

$\mathrm{Au}$ sein de l'anneau de gaz, un certain nombre de condensations de formation stellaire sont observées (objets N, S et SW sur la figure 4) : il s'agit de galaxies naines " recyclées ", en cours de formation dans la matière expulsée du disque de leur galaxie progénitrice. Récemment, on a observé dans ces galaxies naines des vitesses de rotation sur elles-mêmes anormalement élevées [2]. Il est théoriquement aisé de mesurer la masse d'un disque lorsque l'on connaît sa vitesse de rotation, mais l'exercice est bien plus délicat pour ces galaxies naines, encore jeunes et perturbées par leur environnement (noyées dans un anneau de gaz, proches d'une galaxie plus massive), et surtout peu résolues par les télescopes. La confrontation aux modèles numériques (fig. 5) a permis de rétablir les informations manquantes dans les données observationnelles, telles que la forme complète du système et son inclinaison par rapport à notre ligne de visée, rendant possible de mesurer la masse de ces galaxies. Le résultat est d'ailleurs surprenant : la masse de ces galaxies naines " recyclées " se révèle, en effet, notablement supérieure à leur masse visible. Un fait qui serait assez banal pour une galaxie " classique ", la différence entre la masse totale et la masse visible correspondant à la contribution de la célèbre "matière noire ". Mais ces galaxies naines-là sont bien particulières, formées dans les débris arrachés au disque d'une galaxie spirale par une violente collision. Or, les disques des galaxies spirales sont habituellement supposés ne pas contenir de matière noire - laquelle se cacherait plutôt dans un grand halo sphérique entourant le disque.

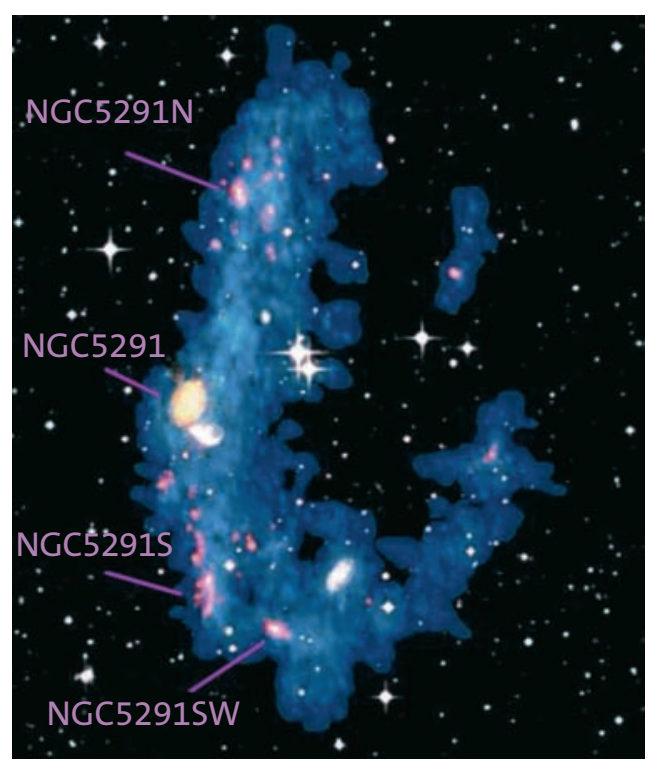

Figure 3 : Simulation numérique de la collision entre les galaxies d'Andromède et M32, donnant naissance aux anneaux concentriques de la galaxie d'Andromède. Ce modèle explique à la fois la morphologie des anneaux et la position actuelle de la galaxie M32, la collision étant survenue presque au centre du disque d'Andromède, il y a 210 millions d'années. Les trois premières images $(a, b, c)$ montrent l'évolution sous l'effet de la collision avec M32 ; le temps est en millions d'années, $t=0$ correspondant à l'instant de la collision et $t=210$ à aujourd'hui. L'image d correspond à l'état actuel de la galaxie d'Andromède si la collision n'avait pas eu lieu: sa structure spirale aurait tourné sur elle-même sans être profondément affectée.

(2) La force de marée est ici la différence entre les forces gravitationnelles subies par deux régions diamétralement opposées d'une même galaxie.

Figure 4 : La galaxie NGC5291 (jaune) et son grand anneau de débris de collision (en bleu : émission de l'hydrogène atomique à 21 centimètres de longueur d'onde). De nouvelles galaxies naines "recyclées". principalement NGC5291-N, S et SW, se forment dans cet anneau de gaz : en rose, l'émission ultraviolette des étoiles jeunes. 

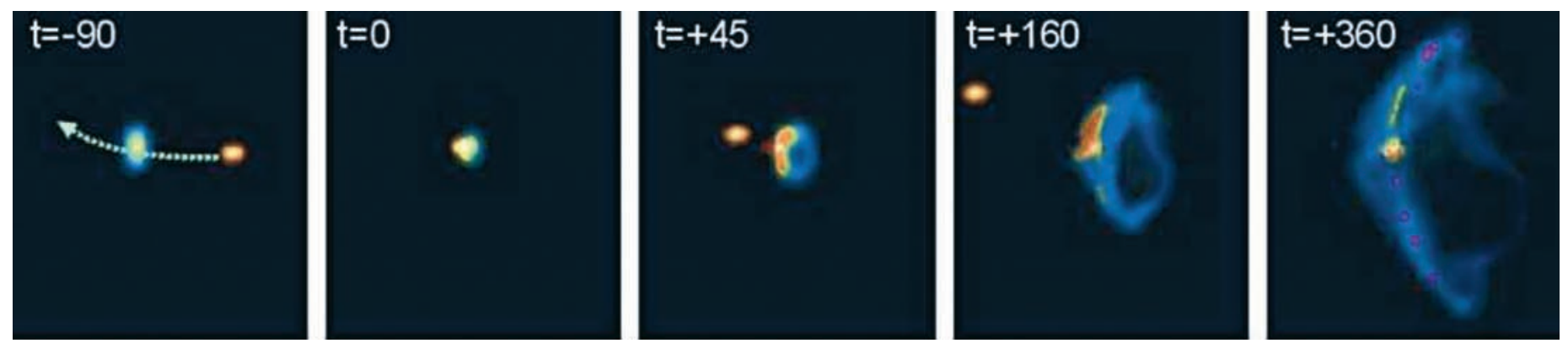

Figure 5 : Simulation numérique de la formation de NGC5291 dans une collision entre deux galaxies massives, il y a 360 millions d'années. Le gaz est en bleu, les étoiles jeunes en rose et les vieilles étoiles en orange. L'échelle de temps est en millions d'années. La galaxie NGC5291, initialement vue sous la forme d'un disque bleuté, est percutée par une galaxie elliptique qui la traverse et qui s'échappe à grande vitesse. Le gaz de son disque est alors expulsé dans un grand anneau de débris de collision, et le centre de la galaxie contenant les vieilles étoiles est transformé en une galaxie elliptique. De nouvelles galaxies naines se créent dans l'anneau, formant de nouvelles étoiles à un rythme élevé. Ce modèle numérique a permis de comprendre les propriétés de ces galaxies naines "recyclées " et la structure du système à trois dimensions, mettant en évidence la présence inattendue de matière noire dans les débris de collision arrachés au disque d'une galaxie.

\section{Références}

[1] Block D., Bournaud F., Combes F. et al., "An almost head-on collision as the origin of two off-centre rings in the Andromeda galaxy", Nature 443 (2006) 832

www.dapnia.cea.fr/Sap/Actualites /Breves/bournaud061019/page. shtml. Ce site comporte un film en français, à haute résolution, téléchargeable (MPG, $25 \mathrm{Mo}$ ).

[2] Bournaud F., Duc P.-A.,

Brinks E. et al., "Missing Mass in Collisional Debris from

Galaxies”, Science 316 (2007)

1166.

www.dapnia.cea.fr/Sap/Actualites /Breves/bournaud070510/page. shtml.

[3] Cox, T. J. \& Loeb, A.

"The Collision between the Milky Way and Andromeda", MNRAS 2007.

www.cfa.harvard.edu/ tcox/ localgroup/.
La masse anormale de ces débris de collisions est alors difficilement explicable si une partie de la matière noire ne se trouve pas elle-même dans le disque de la galaxie spirale progénitrice. C'est là un résultat fondamental pour l'évolution de toutes les galaxies spirales, qui se base avant tout sur des observations, mais qui n'aurait pas pu être mis en évidence sans l'apport crucial des simulations numériques et de leur haute résolution.

\section{Notre Voie Lactée bientôt victime à son tour}

L'histoire tourmentée des galaxies ne s'est pas encore achevée. Notre Voie Lactée fait partie de ces galaxies spirales qui ont eu jusqu'ici une évolution relativement calme. Au cours des huit derniers milliards d'années au moins, elle a certes avalé quelques petites galaxies naines comme elle est en train de le faire encore aujourd'hui avec les nuages de Magellan cependant aucune collision majeure n'est venue bouleverser sa morphologie.

Mais, dans deux milliards d'années, notre galaxie entrera en collision avec la galaxie d'Andromède. Cette collision majeure entre deux galaxies de masses similaires bouleversera complètement les propriétés des deux protago- nistes. La relaxation violente transformera leurs disques spiraux en une galaxie elliptique géante. Des simulations numériques ont là aussi été employées pour étudier l'évolution de cette collision de galaxies [3], qui est un exemple typique d'événements violents tels que la plupart des galaxies en ont déjà connus par le passé. Quant à notre Soleil, il y a des chances importantes qu'il se retrouve expulsé dans le milieu intergalactique par les forces de marée !

Au-delà de cet exemple, les collisions de galaxies ne font pas que façonner des morphologies particulières. Elles sont aussi responsables de la chute de grandes quantités de gaz interstellaire dans les régions centrales des galaxies. Ce gaz, devenu alors très dense, s'effondre sous l'effet de sa gravité à un rythme élevé, en de véritables flambées de formations stellaires. Au moins la moitié des étoiles qui composent aujourd'hui l'Univers seraient nées dans ces flambées. Mais, à ce jour, les modèles théoriques peinent à expliquer comment une galaxie peut subitement se mettre à former des centaines d'étoiles par an, contre moins de dix en temps normal. La compréhension de l'histoire de la formation stellaire reste l'un des problèmes cruciaux de la cosmologie moderne, dont les simulations numériques détiennent peut-être à nouveau l'une des clés.

\section{Les moyens numériques employés}

Modéliser l'évolution d'une galaxie nécessite de simuler l'évolution d'un système d'un ou plusieurs millions de particules sur plusieurs milliers de pas de temps. L'espace est divisé en grilles, généralement de dimensions $256 \times 256 \times 256$ ou $512 \times 512 \times 512$, sur lesquelles est calculé le potentiel gravitationnel. Un ordinateur de bureau aurait besoin de plusieurs semaines pour effectuer une seule simulation, si tant est qu'il dispose d'une mémoire suffisante au stockage de toutes ces données. La modélisation d'un système observé nécessitant généralement d'effectuer plusieurs dizaines de simulations différentes, les scientifiques ont recours aux supercalculateurs parallèles et vectoriels, notamment ceux du CEA et du CNRS. L'évolution d'une galaxie ou d'un système de galaxies en collisions au cours du dernier milliard d'années peut alors être calculée en quelques heures seulement.

Le calculateur utilisé pour ces simulations, de type vectoriel, au CEA/CCRT, possède une puissance-crête de 2,3 Téraflops (un flop = une opération en virgule flottante par seconde, un téra $=$ mille milliards) lorsque tous ses processeurs sont dédiés en même temps a une même simulation. Sa mémoire vive est proche du téraoctet, lui permettant de stocker l'ensemble des variables d'un problème physique complexe. 